\title{
Positional uncertainties of cervical and upper thoracic spine in stereotactic body radiotherapy with thermoplastic mask immobilization
}

\author{
Seung Hyuck Jeon, MD, Jin Ho Kim, MD, PhD \\ Department of Radiation Oncology, Seoul National University Hospital, Seoul, Korea
}

\begin{abstract}
Purpose: To investigate positional uncertainty and its correlation with clinical parameters in spine stereotactic body radiotherapy (SBRT) using thermoplastic mask (TM) immobilization.

Materials and Methods: A total of 21 patients who underwent spine SBRT for cervical or upper thoracic spinal lesions were retrospectively analyzed. All patients were treated with image guidance using cone beam computed tomography (CBCT) and 4 degrees-of-freedom (DoF) positional correction. Initial, pre-treatment, and post-treatment CBCTs were analyzed. Setup error (SE), pre-treatment residual error (preRE), post-treatment residual error (postRE), intrafraction motion before treatment (IM1), and intrafraction motion during treatment (IM2) were determined from 6 DoF manual rigid registration.

Results: The three-dimensional (3D) magnitudes of translational uncertainties (mean \pm 2 standard deviation) were $3.7 \pm 3.5 \mathrm{~mm}$ (SE), $0.9 \pm 0.9 \mathrm{~mm}$ (preRE), $1.2 \pm 1.5 \mathrm{~mm}$ (postRE), $1.4 \pm 2.4 \mathrm{~mm}(\mathrm{IM} 1)$, and $0.9 \pm 1.0 \mathrm{~mm}(\mathrm{IM} 2)$, and average angular differences were $1.1^{\circ} \pm 1.2^{\circ}$ (SE), $0.9^{\circ} \pm 1.1^{\circ}$ (preRE), $0.9^{\circ} \pm 1.1^{\circ}$ (postRE), $0.6^{\circ} \pm 0.9^{\circ}\left(\mathrm{IM} 1\right.$ ), and $0.5^{\circ} \pm 0.5^{\circ}$ (IM2). The 3D magnitude of SE, preRE, postRE, IM1, and IM2 exceeded $2 \mathrm{~mm}$ in $18,0,3,3$, and 1 patients, respectively. No association were found between all positional uncertainties and body mass index, pain score, and treatment location ( $p>0.05$, Mann-Whitney test). There was a tendency of intrafraction motion to increase with overall treatment time; however, the correlation was not statistically significant $(p>0.05$, Spearman rank correlation test).

Conclusion: In spine SBRT using TM immobilization, CBCT and 4 DoF alignment correction, a minimum residual translational uncertainty was $2 \mathrm{~mm}$. Shortening overall treatment time and 6 DoF positional correction may further reduce positional uncertainties.
\end{abstract}

Keywords: Radiosurgery, Radiotherapy, Spine, Neoplasm metastasis, Setup errors

\section{Introduction}

Metastatic cancer to the spine can cause progressive pain, spinal instability, and eventual spinal cord compression that can significantly deteriorate quality of life. For decades, radiotherapy has been used effectively to ameliorate pain and prevent disease progression [1]. With the advent of modern technologies enabling image-guided radiation delivery and steep dose gradient, stereotactic body radiotherapy (SBRT) for spinal lesions became feasible. SBRT can be used to control pain and prevent cord compression in patients with oligometastatic cancer, and local control of spinal tumors after

Received 29 December 2017, Revised 11 February 2018, Accepted 7 March 2018.

Correspondence: Jin Ho Kim, MD, PhD, Department of Radiation Oncology, Seoul National University College of Medicine, 103 Daehak-ro, Jongno-gu, Seoul 03080, Korea. Tel: +82-2-2072-4767, Fax: +82-2-765-3317, E-mail: jinho.kim.md@gmail.com

(c) This is an Open Access article distributed under the terms of the Creative Commons Attribution Non-Commercial License (http://creativecommons.org/ licenses/by-nc/4.0/) which permits unrestricted non-commercial use, distribution, and reproduction in any medium, provided the original work is properly cited.

www.e-roj.org 
SBRT is high as 80\%-90\% [2-4].

In SBRT of spinal tumors, rigorous understanding and minimizing positional uncertainty is critical in order to achieve safe treatment, because inadvertent high doses to spinal cord may result in serious complication [5]. Since the cervical vertebrae and cervico-thoracic junction are the most mobile portion of the spinal column, accurate alignment is especially challenging for SBRT of these spinal locations. In our institution, thermoplastic mask (TM) is used in SBRT for lesions in cervical and upper thoracic spines. TM is a widely used immobilization device for patients with lesions in brain, head-and-neck, and cervical spines. Several researchers have reported the positional errors for mask immobilizers [6-14]. However, different immobilization and analysis methods among researchers complicates consensus on positional uncertainties for spinal SBRT using TM immobilization.

The current study is a retrospective analysis to quantitatively determine and characterize positional uncertainties associated with TM immobilization for spine SBRT.

\section{Materials and Methods}

\section{Patient selection and data collection}

Medical records of patients who underwent SBRT to cervical and upper thoracic spines from December 2014 to July 2017 were retrospectively reviewed. As a 6 degrees-of-freedom (DoF) couch was installed in our institution July 2017, patients treated after this period were excluded. In addition, patients whose treatment violated the procedure described in the following treatment procedure section were excluded from the analysis. The clinical characteristics including pain score and body mass index (BMI) of patients were collected from electronic medical records of our institution. The pain score before treatment was recorded as numeric rating scale with score of 10 to be the maximal pain. Center of treatment, introduced to represent treatment location, was defined as the middle spine level among treated vertebrae. This study was approved by the Institutional Review Board of Seoul National University Hospital (No. 1804-016-934).

\section{Treatment procedure}

Planning CT was acquired using the Brilliance CT Big Bore (Philips, Amsterdam, The Netherlands) with an 1.5-mm slice thickness and TM immobilization device that encompassed the entire head and bilateral shoulders of patients. No bite block was used in junction with TM. An example of patient setup with TM is shown on Fig. 1. Clinical target volume was

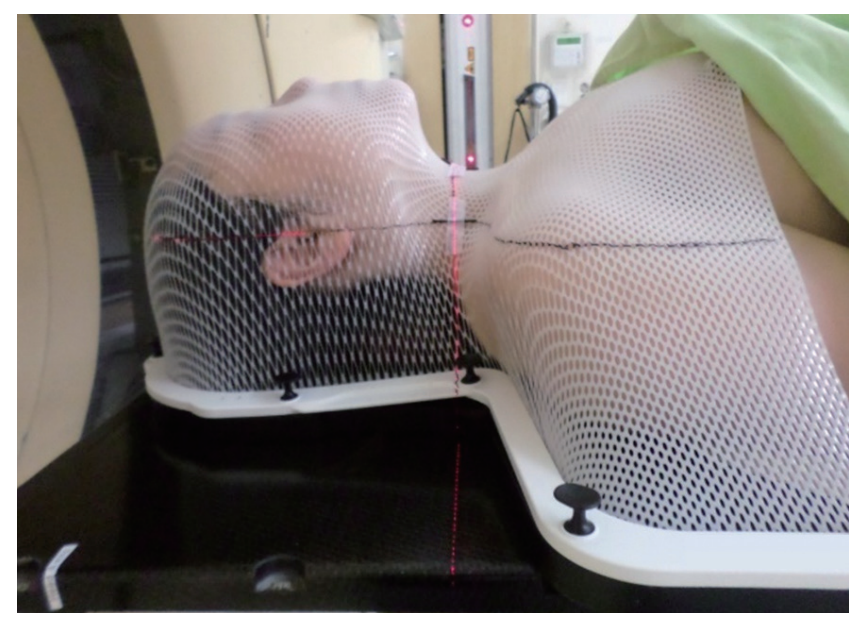

Fig. 1. An example of patient setup at simulation using thermoplastic mask.

delineated according to international consensus guidelines [15]. SBRT plans using volumetric modulated arc therapy (VMAT) technique were generated using the Eclipse system (Varian Medical Systems, Palo Alto, CA, USA). Patients were treated with $10 \mathrm{MV}$ flattening filter-free photon beams using the TrueBeam STx machine (Varian Medical Systems).

For SBRT delivery, patients were set up and immobilized in identical manners as done for planning CT. Then, on-board CBCT was acquired (initial CBCT), and patient alignment was corrected using a $4 \mathrm{DoF}$ couch. The $4 \mathrm{DoF}$ couch is capable of translating through lateral ( $x$, right vs. left), vertical ( $y$, up vs. down), and longitudinal ( $\mathrm{z}$, in vs. out) and rotating around vertical axis (yaw). After every couch movement, verifying CBCT was obtained until no additional movement was needed. If acceptable alignment could not be obtained with repeated 4 DoF couch movement, the patient set up and immobilization was repeated from initial steps. Once alignment was confirmed acceptable in verifying CBCT (pre-treatment CBCT), SBRT was delivered and post-treatment CBCT was obtained immediately after delivery was completed.

\section{Uncertainty quantification}

Simulation $C T$, initial $C B C T$, pre-treatment $C B C T$, and posttreatment CBCT were compared to calculate positional uncertainties. For all comparison, 6 DoF manual rigid registration tool provided by the Eclipse system was used. In patients received multi-fraction SBRT, CBCT images from the first fraction were used for analysis. Five types of positional uncertainties were determined via pairwise comparisons of various CT images: setup error (SE; initial CBCT - simulation 
CT), pre-treatment residual error (preRE; pre-treatment CBCT - simulation CT), post-treatment residual error (postRE; posttreatment $\mathrm{CBCT}$ - simulation $\mathrm{CT}$ ), intrafraction motion during image guidance (IM1; pre-treatment CBCT - initial CBCT), and intrafraction motion during treatment (IM2; post-treatment $\mathrm{CBCT}$ - pre-treatment $\mathrm{CBCT}$ ). For each pairwise comparison, three dimensional (3D) vectors obtained for translational uncertainties along three cardinal directional axes $(x, y, z)$ and rotational uncertainties around those axes, respectively.

Translational components were recorded in positive numbers when errors were in the right, anterior, and inferior directions. Counter-clockwise rotational uncertainties viewed from right (pitch), anterior (yaw), and inferior (roll) sides of the patient was recorded in positive. Magnitude of translational uncertainty $\left(\Delta_{3 D}\right)$ was calculated as

$$
\Delta_{3 \mathrm{D}}=\left(\Delta_{\mathrm{X}^{2}}+\Delta_{\mathrm{y}^{2}}+\Delta_{\mathrm{Z}^{2}}\right)^{1 / 2}
$$

where $\Delta$ is a translational uncertainty and $x, y$, and $z$ correspond to mediolateral, craniocaudal, and anteroposterior components, respectively. As a representative of rotational errors, an average of angular differences $\left(\theta_{\text {avg }}\right)$ proposed by Tryggestad et al. [7] was defined as

$$
\theta_{\text {avg }}=\left(\left|\theta_{\text {pitch }}\right|+\left|\theta_{\text {roll }}\right|+\left|\theta_{\text {yaw }}\right|\right) / 3
$$

where $\theta$ is a rotational uncertainty around three cardinal axes.

\section{Statistical analyses}

All quantitative variables were expressed as mean \pm 2

Table 1. Patient and treatment characteristics

\begin{tabular}{lc}
\hline \multicolumn{1}{c}{ Characteristic } & Value \\
\hline Location & $14(66.7)$ \\
C-spine & $7(33.3)$ \\
T-spine & \\
Dose & $5(23.8)$ \\
$16 \mathrm{~Gy} / 1 \mathrm{fx}$ & $10(47.6)$ \\
$18 \mathrm{~Gy} / 1 \mathrm{fx}$ & $3(14.3)$ \\
$20 \mathrm{~Gy} / 1 \mathrm{fx}$ & $1(4.8)$ \\
$22 \mathrm{~Gy} / 1 \mathrm{fx}$ & $1(4.8)$ \\
$24 \mathrm{~Gy} / 1 \mathrm{fx}$ & $1(4.8)$ \\
$24 \mathrm{~Gy} / 3 \mathrm{fx}$ & $22.75(16.94-28.72)$ \\
BMI (kg/m $\left.{ }^{2}\right)$ & $4.5(0-8)$ \\
Pain score & $4(3-7)$ \\
Number of CBCTs & \\
\hline
\end{tabular}

Values are presented as number (\%) or median (range).

$\mathrm{BMI}$, body-mass index; $\mathrm{CBCT}$, cone-beam computed tomography. standard deviation (SD). We used the Shapiro-Wilk test to check normality of all variables. The $\Delta_{3 \mathrm{D}}$ and $\theta_{\text {avg }}$ of different uncertainties were compared with Wilcoxon signed-rank test. Comparisons of SD between the three translational or three rotational components within a given uncertainty were performed using Levene test. The correlation between clinical characteristics and uncertainties were evaluated with MannWhitney test and Spearman rank correlation test. A p-value less than 0.05 was considered statistically significant. All statistical analyses were performed using $R$ software (version 3.4.0; R Foundation for Statistical Computing, Vienna, Austria).

\section{Results}

\section{Patient and treatment characteristics}

Total of 21 patients were included in the current analysis. The characteristics of included patients and treatments are detailed on Table 1. The lowest level of treated spine was T5 vertebra. The median number of obtained CBCT per treatment (post-treatment CBCT included) was 4 (range, 3 to 7). The most commonly used dose schedule was 18 Gy in 1 fraction. The median value of pain score and BMI were 4.5 (range, 0 to 8) and $22.75 \mathrm{~kg} / \mathrm{m}^{2}$ (range, 16.94 to $28.72 \mathrm{~kg} / \mathrm{m}^{2}$ ). The center of treatment was cervical and thoracic spines in 14 and 7 patients, respectively.

\section{Positional uncertainties}

Positional uncertainties are demonstrated on Table 2. Significant number of these uncertainties did not conform to a normal distribution ( $p<0.05$ on Shapiro-Wilk test). Among translational uncertainties, SE showed the greatest $\Delta_{3 \mathrm{D}}(3.7 \pm 3.5$ $\mathrm{mm})$. The $\Delta_{3 D}$ of postRE $(1.2 \pm 1.5 \mathrm{~mm})$ was significantly greater than that of preRE $(0.9 \pm 0.9 \mathrm{~mm})$. Also, $\Delta_{3 \mathrm{D}}$ of IM $1(1.4 \pm 2.4$ $\mathrm{mm})$ was significantly greater than that of IM2 $(0.9 \pm 1.0 \mathrm{~mm})$ In all 21 patients, $\Delta_{3 D}$ for $S E$, preRE, postRE, IM1, and IM2 exceeded $2 \mathrm{~mm}$ in 18 (86\%), 0 (0\%), 3 (14\%), 3 (14\%), and 1 $(5 \%)$ patients, respectively. The distributions of $\Delta_{3 D}$ for residual errors and intrafraction motions are demonstrated on Fig. 2. In terms of rotational uncertainties, $\theta_{\text {avg }}$ of $\operatorname{IM} 1\left(0.6^{\circ} \pm 0.9^{\circ}\right)$ and $\operatorname{IM} 2\left(0.5^{\circ} \pm 0.5^{\circ}\right)$ were significantly less than SE $\left(1.1^{\circ} \pm 1.2^{\circ}\right)$ preRE $\left(0.9^{\circ} \pm 1.1^{\circ}\right)$, and postRE $\left(0.9^{\circ} \pm 1.1^{\circ}\right)$. There was no significant difference in $\theta_{\text {avg }}$ between preRE and postRE or between IM1 and IM2.

Among rotational uncertainties of preRE and postRE, SD of a yaw component was significantly smaller than that of roll and pitch errors (Levene test). There was no difference in SD between translational components of respective uncertainties. 


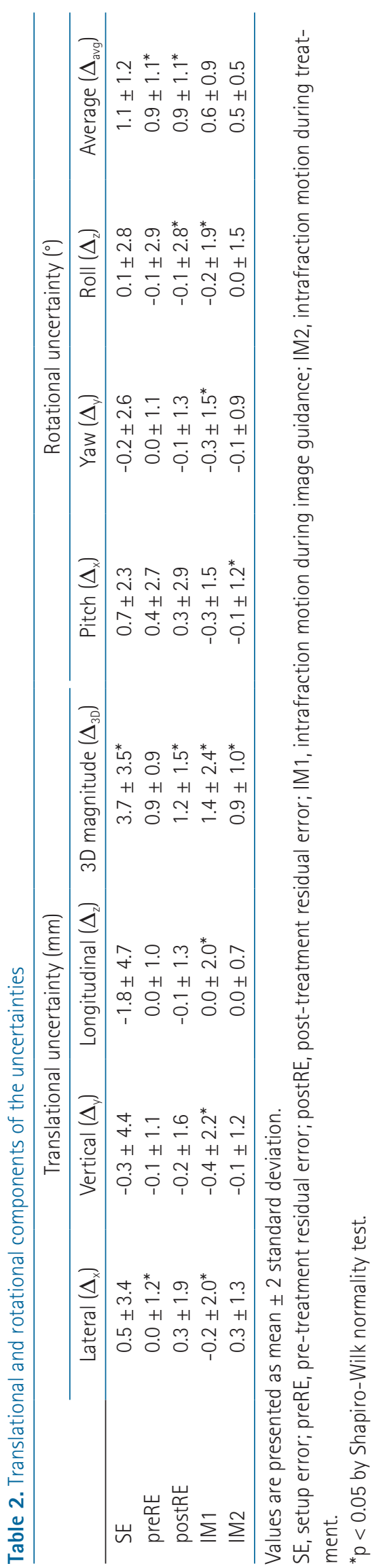

A

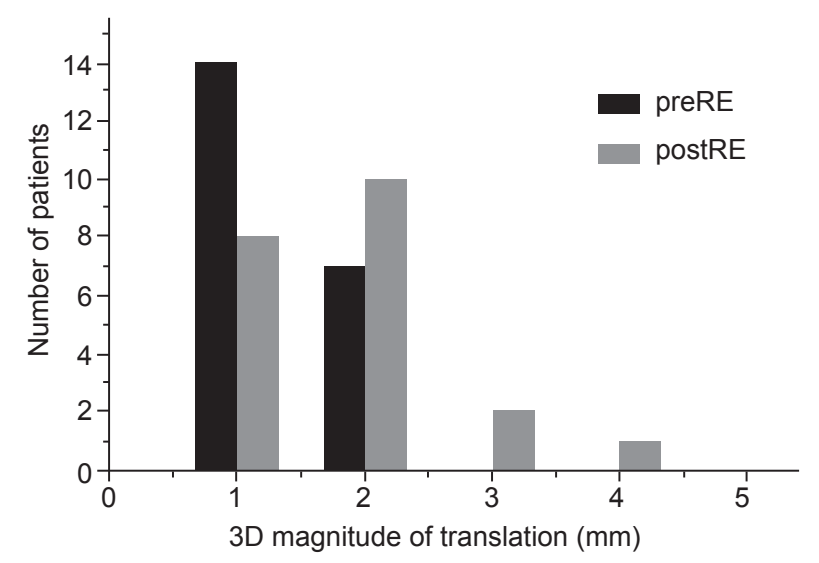

B

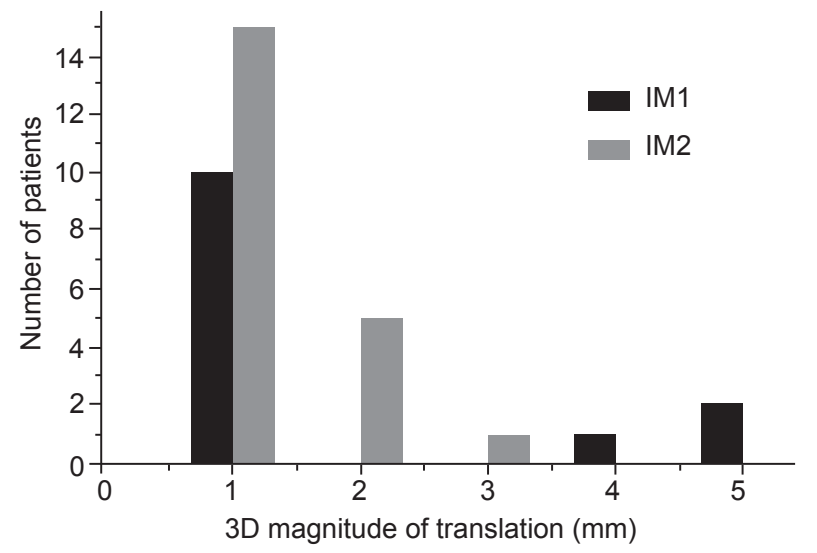

Fig. 2. Histogram of three-dimensional (3D) magnitude of translation $\left(\Delta_{30}\right)$ of preRE and postRE $(A)$, and IM1 and IM2 (B) in 21 patients. preRE and postRE represent pre- and post-treatment residual error; IM1 and IM2, intrafraction motion during image guidance and during treatment.

\section{Association with clinical factors}

The 20 patients with their BMI available were dichotomized into high ( $\left.>23 \mathrm{~kg} / \mathrm{m}^{2}, \mathrm{n}=10\right)$ and low BMI $\left(\leq 23 \mathrm{~kg} / \mathrm{m}^{2}, \mathrm{n}\right.$ $=10)$ group. There was no difference in $\Delta_{30}$ and $\theta_{\text {avg }}$ in the two groups. Among 18 patients with available pain scores, 9 patients with pain score $\geq 5$ showed no different $\Delta_{3 \mathrm{D}}$ and $\theta_{\text {avg }}$ than remaining 9 patients whose pain score was less than 5. In addition, there was no correlation between treatment site (cervical spines vs. thoracic spines) and any uncertainties (Mann-Whitney test).

Next, a correlation of magnitudes of intrafraction motions with time intervals between CBCT acquisitions was analyzed. Median time intervals of CBCT were 805 seconds (range, 354 to 1,384 seconds) and 366 seconds (range, 290 to 587 seconds) for IM1 and IM2, respectively. The correlation between imaging 
acquisition intervals and intrafraction motions, combining IM1 and $\mathrm{IM} 2$, is shown on Fig. 3. We could not find the association of CBCT interval and 3D magnitude nor average angular differences (all $p>0.05$, Spearman rank correlation test), though there was a tendency of uncertainty to increase as interval extends.

\section{Discussion and Conclusion}

We comprehensively described various positional uncertainties encountered in SBRT of cervical and upper thoracic spines aided by TM immobilization, CBCT image guidance, and 4 DoF couch. Positional uncertainties in spines immobilized by TM have been reported by several researchers. Murphy et al. [12] reported that short-term motion of TM-immobilized head and spine was within $0.8 \mathrm{~mm}$ using two orthogonal x-ray views. Li et al. [16], based on 18 patients receiving spinal SBRT delivered using TM, reported that 3-mm margin was required to encompass total uncertainty, and that SD of interfraction SE ranged from 2 to $3 \mathrm{~mm}$. According to Ohtakara et al. [11], the maximal translational REs were less than $2 \mathrm{~mm}$ for conventional TM. The current study showed that 2SD of translational uncertainties or $\Delta_{3 \mathrm{D}}$ did not exceed 2 $\mathrm{mm}$ for preRE, postRE, and IM2 in all patients. These findings are generally in accordance with previous reports, and suggest that a 2-mm planning target volume (PTV) margin is appropriate for SBRT of cervical and upper thoracic spines immobilized with TM.

The current study found no significant association of clinical characteristics, including $\mathrm{BMI}$, treatment location, and pain score with positional uncertainties. Although not significantly associated, there was a trend that translational and angular uncertainties increased as longer time intervals elapsed. In our series, treatment was less than 10 minutes in all patients. A median interval between pre-treatment and post-treatment CBCT was 6 minutes 6 seconds. This fast treatment delivery was achieved by VMAT techniques and flattening filter-free beam, and likely contributed to a small intrafraction motion [17].

Our findings showed the potential impact of couch motion functionality on positional errors. Patients in the current series were aligned on the 4 DoF couch, which is able to correct translational errors and yaw differences. There was no correcting roll and pitch rotations. In both preRE and postRE, yaw rotational uncertainties were significantly smaller than those of pitch and roll. This was because angular errors around the yaw axis was partly corrected using the 4 DoF couch. To the contrary, there was no difference in three components of rotational uncertainties in $\mathrm{SE}_{1} \mathrm{IM} 1$, and IM2, for they were completely random. These findings show that a couch of 6 DoF motion functionality may further likely reduce angular uncertainties.

Assuming the perfect pre-treatment setup using the 6 DoF couch, translational and angular preRE will be zero. However, it is impossible to eliminate intrafraction motion. Thus, in the above ideal setup situation, postRE should be exactly equal to IM2. Our analysis showed that postRE was significantly greater than IM2, because the 4 DoF couch was unable to eliminate preRE. Thus, compared with the 4 DoF counterpart, the 6 DoF might further reduce minimal PTV margins to adequately address postRE.

Findings from the current study may be useful in

A

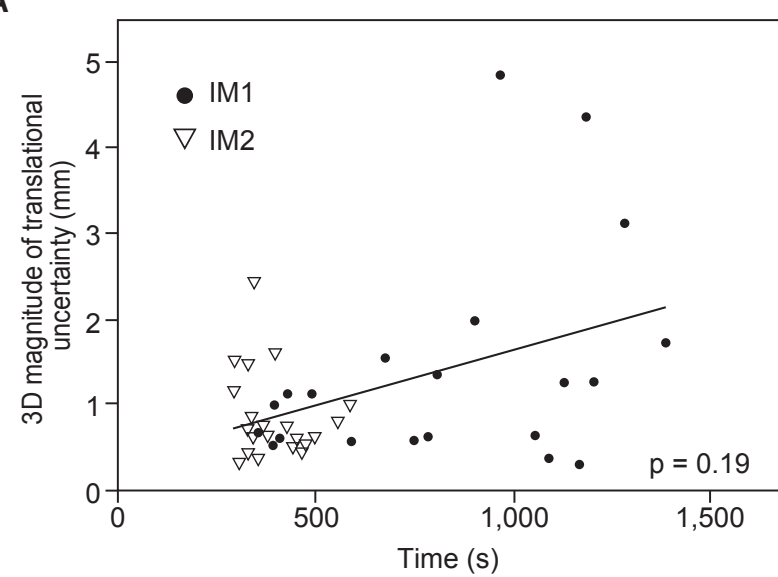

B

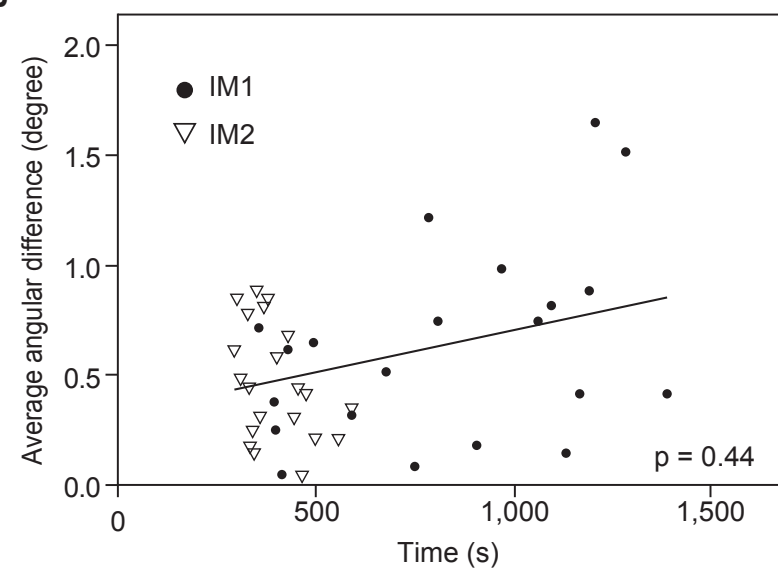

Fig. 3. Correlation of $(A) 3 D$ magnitude $\left(\Delta_{3 D}\right)$ of translation and (B) average angular difference $\left(\theta_{\text {avg }}\right)$ with time intervals between CBCT acquisitions. Solid lines represent linear regression lines; IM1 and $I M 2$, intrafraction motion during image guidance/treatment; $\mathrm{CBCT}$, cone-beam computed tomography. 
determining PTV margins for frameless stereotactic radiosurgery of intracranial lesions, because skull and cervical spines were immobilized by TM. Several researchers reported the positional errors of frameless radiosurgery of intracranial lesions. Rosenfelder et al. [13] prospectively investigated the uncertainties using frame-based immobilization and TM. They concluded that fixation using TM with daily online correction provides similar uncertainty compared with frame-based immobilization. Ramakrishna et al. [14] reported smaller intrafraction motion with frame-based radiotherapy, but intrafraction motion with TM was also clinically acceptable with mean value of $0.7 \mathrm{~mm}$. In addition, Gevaert et al. [10] showed that frameless immobilization device for intracranial radiosurgery had the positional uncertainty of less than 1 $\mathrm{mm}$, and suggested the minimum safety margin of $2 \mathrm{~mm}$. Considering there is no movable joints in the skull, it may be safely assumed that any positional uncertainties of the skull and intracranial structures are not greater than those of cervical and upper spines. Thus, based on our data, we propose a 2-mm PTV margin for intracranial radiosurgery using TM immobilization.

The current study was limited by a relatively small number of analyzed patients. Many uncertainty components were found to show non-normal distribution. Whether those positional errors are inherently non-normal was not to be decided due to limited number of observations.

In summary, TM immobilization combined with CBCT image guidance and 4 DoF alignment correction provide adequate positioning for patients receiving SBRT for cervical and upper thoracic lesions. A minimum residual translational uncertainty of 2-mm should be considered in relations with both clinical target volumes and organs at risk. Though eliminable, intrafraction motion may be minimized by reducing overall treatment time. The couch with 6 DoF motion functionality has a potential to further reduce positional uncertainties by minimizing pre-treatment angular residual errors.

\section{Conflict of Interest}

No potential conflict of interest to this article was reported.

\section{References}

1. Chow $E$, Zeng L, Salvo N, Dennis K, Tsao M, Lutz S. Update on the systematic review of palliative radiotherapy trials for bone metastases. Clin Oncol (R Coll Radiol) 2012;24:112-24.

2. Greco $C_{1}$ Pares 0 , Pimentel $N$, et al. Spinal metastases: from conventional fractionated radiotherapy to single-dose SBRT Rep Pract Oncol Radiother 2015;20:454-63.

3. Ryu S, Yoon H, Stessin A, Gutman F, Rosiello A, Davis R. Contemporary treatment with radiosurgery for spine metastasis and spinal cord compression in 2015. Radiat Oncol J 2015;33:1-11.

4. Ryu S, Pugh SL, Gerszten PC, et al. RTOG 0631 phase 2/3 study of image guided stereotactic radiosurgery for localized (1-3) spine metastases: phase 2 results. Pract Radiat Oncol 2014;4:76-81.

5. Grimm J, Sahgal A, Soltys SG, et al. Estimated risk level of unified stereotactic body radiation therapy dose tolerance limits for spinal cord. Semin Radiat Oncol 2016;26:165-71.

6. Qi XS, Hu AY, Lee SP, et al. Assessment of interfraction patient setup for head-and-neck cancer intensity modulated radiation therapy using multiple computed tomography-based image guidance. Int J Radiat Oncol Biol Phys 2013;86:432-9.

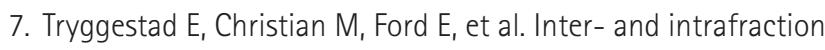
patient positioning uncertainties for intracranial radiotherapy: a study of four frameless, thermoplastic mask-based immobilization strategies using daily cone-beam CT. Int J Radiat Oncol Biol Phys 2011;80:281-90.

8. van Kranen S, Mencarelli A, van Beek S, Rasch C, van Herk M, Sonke JJ. Adaptive radiotherapy with an average anatomy model: evaluation and quantification of residual deformations in head and neck cancer patients. Radiother Oncol 2013;109:463-8.

9. Jeon W, Wu HG, Song SH, Kim Jl. Radial displacement of clinical target volume in node negative head and neck cancer. Radiat Oncol J 2012;30:36-42.

10. Gevaert T, Verellen D, Tournel K, et al. Setup accuracy of the Novalis ExacTrac 6DOF system for frameless radiosurgery. Int $\rfloor$ Radiat Oncol Biol Phys 2012;82:1627-35.

11. Ohtakara K, Hayashi S, Tanaka H, et al. Clinical comparison of positional accuracy and stability between dedicated versus conventional masks for immobilization in cranial stereotactic radiotherapy using 6 -degree-of-freedom image guidance system-integrated platform. Radiother Oncol 2012;102:198205.

12. Murphy MJ, Chang SD, Gibbs IC, et al. Patterns of patient movement during frameless image-guided radiosurgery. Int J Radiat Oncol Biol Phys 2003;55:1400-8.

13. Rosenfelder NA, Corsini L, McNair H, et al. Comparison of setup accuracy and intrafraction motion using stereotactic frame versus 3-point thermoplastic mask-based immobilization for fractionated cranial image guided radiation therapy. Pract Radiat Oncol 2013;3:171-9. 
14. Ramakrishna N, Rosca F, Friesen $S_{1}$ Tezcanli $E_{1}$ Zygmanszki $P_{1}$ Hacker F. A clinical comparison of patient setup and intrafraction motion using frame-based radiosurgery versus a frameless image-guided radiosurgery system for intracranial lesions. Radiother Oncol 2010;95:109-15.

15. Cox BW, Spratt DE, Lovelock $M$, et al. International Spine Radiosurgery Consortium consensus guidelines for target volume definition in spinal stereotactic radiosurgery. Int J
Radiat Oncol Biol Phys 2012;83:e597-605.

16. Li W, Sahgal A, Foote M, Millar BA, Jaffray DA, Letourneau D. Impact of immobilization on intrafraction motion for spine stereotactic body radiotherapy using cone beam computed tomography. Int J Radiat Oncol Biol Phys 2012;84:520-6.

17. Sapkaroski D, Osborne $C$, Knight KA. A review of stereotactic body radiotherapy: is volumetric modulated arc therapy the answer? J Med Radiat Sci 2015;62:142-51. 